\title{
SPATIO-TEMPORAL STORY MAPPING ANIMATION BASED ON STRUCTURED CAUSAL RELATIONSHIPS OF HISTORICAL EVENTS
}

\author{
Yasushi Inoue*, Ken’ichi Tsuruoka and Masatoshi Arikawa \\ Center for Spatial Information Science, The University of Tokyo \\ 5-1-5, Kashiwanoha, Kashiwa City, Chiba 277-8568, Japan - yasushi @ csis.u-tokyo.ac.jp
}

KEY WORDS: History Learning, E-learning, Story visualization, Timelines, Ubiquitous mapping, Push style interfaces

\begin{abstract}
:
In this paper, we proposed a user interface that displays visual animations on geographic maps and timelines for depicting historical stories by representing causal relationships among events for time series. We have been developing an experimental software system for the spatial-temporal visualization of historical stories for tablet computers. Our proposed system makes people effectively learn historical stories using visual animations based on hierarchical structures of different scale timelines and maps.
\end{abstract}

\section{INTRODUCTION}

In history learning, it is important for users to understand effectively causal relationships of events. However, paper textbooks have a limitation of dynamically representing historical stories, because articles of paper textbooks consist of pieces of texts and pictures such as photos, maps, diagrams, and chronological tables. These kinds of articles are fragmental and static descriptions from the viewpoint of visual presentations. For resolving the limitation of paper textbooks, we propose a new framework for dynamic visualization of synchronizing maps and timelines on tablet computers for learners to efficiently understand historical stories with causal relationships of events.

\section{RELATED WORK}

The main purpose of historical learning is to hold future perspectives for our activities through studying historical facts and leveraging acquired knowledge. A history is composed of events based on temporal and causal relationships to describe a theme. It is important for learners to understand causal relationships among historical events written in their explanations in history learning. There are existing software systems (Hara, 2009) for visualizing temporal, spatial relationships and important levels in historical events, however their functions of supporting historical learning are not enough because they were designed for analyzing spatial aspects of temporal information and for managing and retrieving historical materials.

timemap (2012) was created as a software system to visualize historical facts on chronological tables and maps. timemap can visualize events as icons on chronological tables and maps. Users can get the information of events by clicking the icons on them. One of weak points of timemap is that it provides all information on one layers of a chronological table and a map which are too much for a user to understand causal relationships through a large amount of data.

We proposed a new framework and are developing a software system on it to provide users with appropriate amount of historical information from a large amount of historical events related with a certain theme through push style interfaces based on hierarchical structures of historical data with temporal, spatial, causal and semantic relationships. The framework is expected for users to easily and rightly understand causal relationships among historical events.

\section{MULTIPLE TIMELINES}

Events are represented as segments and points on a time axis. A timeline is composed of events in temporal order. When we think about a certain theme in history, multiple timelines should be used to describe it from different viewpoints. Conventional chronological tables are static and usually represent only one timeline because of the limitation of a physical paper. We propose a new method of using multiple timelines and animation-based focal point movement for dynamically representing scales of events. It allows users to learn histories efficiently.

\subsection{Hierarchical Structure of Timelines}

Timelines are represented in the form of hierarchies like zooming functions of web mappings (Figure 1). We focus on a certain event on a timeline. Detailed timelines of a timeline can be available through animations and iterations on interfaces of timelines and maps.

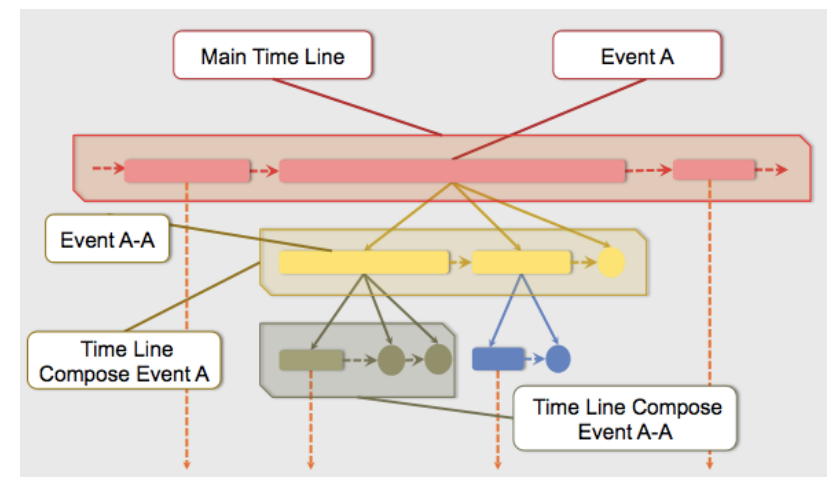

Figure 1. Examples of hierarchical structures of nested timelines. Timelines are composed of events and relationships among them. 


\subsection{Animation as a Traverse on a Hierarchy of Timelines}

An animation is used as a presentation of a story telling events and causal relationships among them. Events exist on different levels of timelines in a hierarchy of them. The story is defined as a path of traversing the hierarchy (Figure 2).

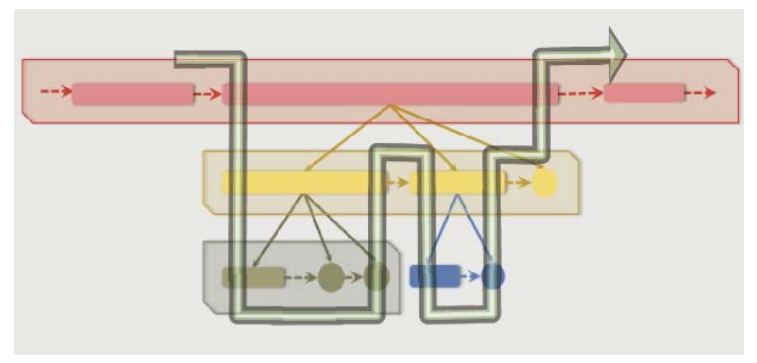

Figure 2. An example of a path of traversing a hierarchy of different detailed timelines. The path becomes a script code of an animation of historical storytelling.

\section{PROTOTYPE}

We have developed a new framework for visualization of historical stories based on causal relationships of events. A historical story can be defined what to combine causal relationships of events along the axis of time. We classified and defined simple data models for visualization according as time series and locations of events. We have implemented an application software system that has an interactive user interface by displaying sequences of graphics with our data models. Our user visual interfaces are realized by three basic methods for depicting historical stories, which will be explained in the following subsections.

\subsection{Visualization of causal relationships with arrow icons on maps and timelines}

All of events occur along time series in history learning. A representation of chronological tables of paper textbooks, however, does not express clearly relationships among each of events. Our proposed system extracts one story sequence from multiple events along time series and causal relationships. A user interface of our system consists of three main views on one screen for visual representations (Figure 3 ). The first main view is a map view, which displays relationships of locations about events. The Second main view is a timeline view, which shows both absolute time of events and orders of events. Third main view will display articles, texts, photos explaining events. Events in three main views are synchronized to each other according as a main story sequence. Orders of events are illustrated with animations of arrow icons on maps. Directions of these arrows depend on relationships among historical events on a subject of a main story sequence. Besides, colors of arrows are decided depending on same categorized events.

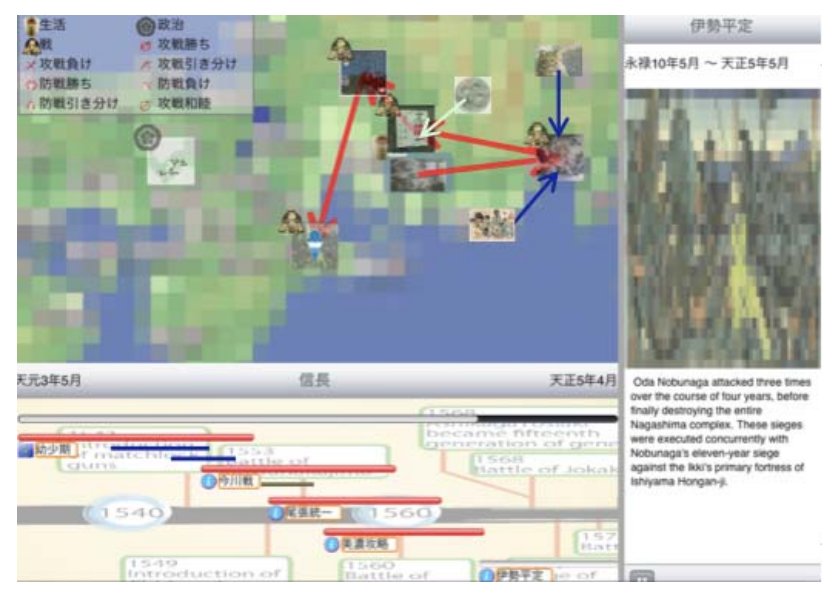

Figure 3. A user interface in a historical story visualization system is composed of displays of maps, timelines and articles.

\subsection{Visualization of hierarchies of events with maps and timelines}

According as time scale, a chronological table of a paper textbook does not have enough physical spaces, if an editor describes the details in one page. In a similar case, representations on locations of events also have limitations in maps of paper textbooks. From the reason, we created a hierarchy structure with a parameter of importance about events. Our system has multiple maps for synchronizing scaled timelines for visualization of these hierarchies.

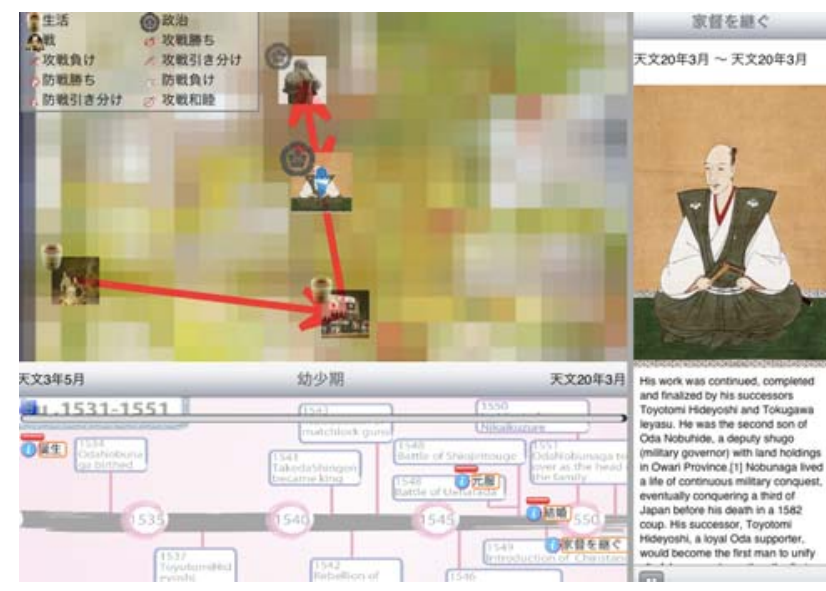

Figure 4. A detailed timeline can be presented after visiting the timeline in Figure 3. A picture and the description of Nobunaga Oda in this figure are used from Wikipedia.

\subsection{Visualization of the focal position in storytelling}

Our proposed system provides users with an interactive and animation-based timelines and maps. Animations of these views are automatically showed depending on a focal position of a main story sequence. The focal position of maps and timelines are synchronized to subjects of a historical story. A focal position changes scale of timelines and maps when a historical hierarchy of a main story sequence changes. Besides, a subject of a focal position shows a set of information consisting of texts, pictures and audios. Users can access linked events and change a main story sequence when users want to go to a next or previous event. 
Rectangles and circles denotes events in Figure 1. Every event is connected to other points. An arrow expresses one causal relationship with another event. Some events can have child events. These events make a hierarchy on time series. A historical story event consists of relationships of sequences. Our proposed system can show historical stories by introducing multiple maps and scaled timelines based on our data model.

The user interface consists of three main views, which are a map, a timeline and an article of a user's current position. Sequences of arrow icons on a map illustrate order and sequence of events for time series. Lines for events on a timeline are synchronized with arrows for events on a map and an article of the current position in the right view. Movement of a focal position can automatically change the scale of timelines and maps when hierarchy levels are changed.

\section{CONCLUSION}

This paper aimed to spatio-temporal visualization of storytelling about historical events. Specifically, our data models, which have arrows and hierarchies of maps and timelines, can provide the easy way of creating animation presentations for history learning. We have proposed a new framework to support users' learning historical stories. In the future work, we will evaluate levels of users' understanding and interesting after they used our proposed system. Furthermore, we are focusing on improving our proposed system for not only visualization of historical stories, but also development of an application software for creating and sharing user-generated content on history study.

\section{References}

Hara, S., 2009. Humanities GIS in Japan: Current Status, Models and Tools. GIS in the Humanities and Social Sciences 2009.

timemap, 2012. Javascript library to help use a SIMILE timeline with online maps including Google, OpenLayers, and Bing. http://code.google.com/p/timemap/ (23 Mar. ,2014). 